\title{
Serie de casos: colgajo sóleo-gastrocnemio en pacientes con defectos de partes blandas en miembros inferiores
}

\author{
Case series: soleo-gastrocnemius muscle flap in patients with defects of soft tissue-lower limbs
}

José Nicolás Rodríguez Lara ${ }^{10}$ https://orcid.org/0000-0003-3953-4678

Mirna Patricia Ochoa Fletes 10 https://orcid.org/0000-0002-5317-3293

Secretaría de Salud Honduras, Hospital Escuela, Servicio de Ortopedia y Traumatología; Tegucigalpa, Honduras.

\begin{abstract}
RESUMEN. Antecedentes: La exposición ósea puede ser consecuencia de fracturas traumáticas abiertas o cerradas con complicaciones, así como secundarias a procesos infecciosos. La cobertura del miembro inferior con defecto cutáneo se basa en la preservación de la perfusión sanguínea y nerviosa para favorecer la consolidación ósea y así el salvamento del miembro. Descripción de los casos: Se describe la reconstrucción con colgajo muscular rotacional pediculado de sóleo y/o gastrocnemio en 16 pacientes con complicaciones asociadas a fracturas expuestas en miembros inferiores con defectos de partes blandas y pérdida de cobertura cutánea, realizados en el Servicio de Ortopedia y Traumatología del Hospital Escuela (HE) durante el período 2012-2015. Se realizó colocación de colgajo con sóleo en $50.0 \%(8 / 16)$ y gastrocnemio en $50.0 \%(8 / 16)$. El éxito terapéutico calificado quirúrgica y funcionalmente fue del $87.5 \%$ (14/16). Conclusión: Con la técnica de reconstrucción realizada a estos pacientes se logró disminuir la frecuencia de complicaciones y por consiguiente evitar la amputación de la extremidad afectada.

Palabras clave: Colgajos quirúrgicos, Honduras, Músculos, Ortopedia.
\end{abstract}

Recibido: 09-08-2019 Aceptado: 14-09-2021 Primera vez publicado en línea: 08-11-2021 Dirigir correspondencia a: Dr. José Nicolás Rodríguez Lara Correo electrónico: jose.rodriguez.85@hotmail.com

DECLARACIÓN DE RELACIONES Y ACTIVIDADES FINANCIERAS Y NO FINANCIERAS: Ninguno

\section{DECLARACION DE CONFLICTOS DE INTERÉS: Ninguno}

Forma de citar: Rodríguez Lara JN, Ochoa Fletes MP. Serie de casos: colgajo sóleogastrocnemio en pacientes con defectos de partes blandas en miembros inferiores. Rev Méd Hondur. 2021; 89(2): 131-135. DOI: https://doi.org/10.5377/rmh.v89i2.12774

(C) 2021 Autor(es). Artículo de acceso abierto bajo la licencia https://creativecommons.org/ licenses/by/4.0/deed.es (c) (i)

\section{INTRODUCCIÓN}

La exposición ósea puede ser consecuencia de fracturas traumáticas abiertas o cerradas, así como secundarias a procesos infecciosos. La cobertura adecuada de la pierna con defecto cutáneo es esencial en el tratamiento del paciente con exposición ósea; uno de los recursos más utilizados en ambientes de bajo presupuesto es el colgajo muscular rotacional pediculado de sóleo y/o gastrocnemio para la reconstrucción del tercio proximal de la pierna, técnica quirúrgica reconstructiva desarrollada en el siglo XIX y cuyos principios básicamente se relacionan con la preservación de la perfusión sanguínea, nerviosa y consolidación ósea cuyo fin último es el salvamento del miembro. ${ }^{1-3}$

La reconstrucción de tejidos blandos de la extremidad está determinada por factores como el estado del paciente y de la herida, el tipo de fractura, estructuras expuestas y riesgo de infección; el objetivo del manejo está en lograr la estabilización ósea y la reconstrucción del tejido blando; sin embargo, es importante obtener una herida limpia libre de tejido contaminado que inhiba la cicatrización; por lo que la limpieza y desbridamiento es necesario. ${ }^{4}$

Entre las ventajas de la reconstrucción están la facilidad de implementación técnica, buenos resultados y buena reproducibilidad en manos de cirujanos ortopédicos; ${ }^{2,5}$ con lo que se logra mejorar la vascularización de la herida y limitar la colonización e infección bacteriana, así también promueven el depósito rápido de colágeno y regeneración tisular temprana. ${ }^{6}$ Las indicaciones principales se asocian con trauma extenso del tercio medio y proximal de la pierna hasta el tercio distal de la cara posterior del muslo y fosa poplítea, defectos de cobertura en artroplastias de rodilla, reconstrucción de tendón patelar o cuadriscipital, pseudoartrosis y contractura de Volkman. Otras indicaciones son las fracturas expuestas con defecto cutáneo que no han podido cerrar por primera intención, defectos por osteomielitis crónica, pseudoartrosis infectadas, pseudoartrosis con fístula cutánea o necrosis de piel. . $2,7,8^{2}$

El procedimiento o técnica quirúrgica en general se realiza colocando un colgajo muscular rotacional pediculado de sóleo y/o gastrocnemio según la indicación y contexto clínico del pa- 
ciente, y posteriormente al verificar la supervivencia de éste se coloca el injerto cutáneo. ${ }^{9}$

En Honduras no hay publicaciones, ni casos reportados sobre la experiencia de la reconstrucción con colgajos musculares rotacionales pediculados en pacientes con fracturas expuestas de origen traumático en miembros inferiores. En el $\mathrm{HE}$ a pesar de que existe una experiencia acumulada de varios años por el servicio de Cirugía Plástica y Reconstructiva, y más recientemente por el Servicio de Ortopedia y Traumatología, no se dispone de información clínico-evolutiva al respecto y dadas las ventajas en cuanto a este procedimiento, que resulta técnicamente reproducible, con recursos accesibles, de bajo costo, con una curva de aprendizaje relativamente corta y con resultados excelentes en la mayoría de los casos, así como mínimas complicaciones, consideramos necesario documentar los resultados de esta experiencia para promover otros estudios de investigación con un mayor número de casos, siendo el objetivo de este reporte describir una serie de casos de reconstrucción con colgajo muscular de sóleo y/o gastrocnemio en pacientes con defectos de partes blandas de miembros inferiores llevado a cabo en el Servicio de Ortopedia y Traumatología del HE, en el período comprendido de 2012 al 2015.

\section{DESCRIPCIÓN DE CASOS}

Se describe una serie de 16 casos de reconstrucción con colgajo muscular de sóleo y/o gastrocnemio en pacientes con defectos de partes blandas de miembros inferiores.

La edad promedio de los pacientes fue 38 años, con una desviación estándar (DE) +/-13.5, del sexo masculino 87.5\% (14) y procedentes del ambiente urbano de Francisco Morazán $75.0 \%$ (12) (Cuadro 1). El 81.1\% (13) no presentaron antecedentes personales patológicos. Los factores de riesgo encontrados al fracaso en la supervivencia del colgajo fueron bocio, hipertensión arterial, diabetes mellitus, con 1 caso respectivamente $6.3 \%$ (1); tenían hábitos tóxicos de alcoholismo y tabaquismo $12.5 \%$ (2), así como condición vascular excelente en $93.7 \%$ (15) (Cuadro 2). Entre las características clínicas el $68.7 \%$ (11) presentó fractura expuesta proximal de tibia; siendo el mecanismo de fractura en $81.3 \%$ (13) secundario a politraumatismo (Cuadro 3). El 93.7\% (15) de las fracturas se manejó de forma abierta con fijador externo tipo Taylor, pseudoartrosis se presentó en el $100.0 \%$ (16). De los 16 casos en el $50.0 \%$ (8) se realizó reconstrucción con colgajo muscular sóleo versus $50.0 \%$ (8) reconstrucción con colgajo muscular gastrocnemio (Figura 1). La tasa de fracaso fue de 6.3\% (1/16). Este caso fue el de un paciente masculino, agricultor de 57 años, sin factores de riesgo y con fractura Grado IIIB con pérdida cutánea, que fue sometido inicialmente a fijación externa y complicándose con pseudoartrosis infectada y pérdida cutánea; a los 8 días se procedió a colocación de colgajo de gastrocnemio medial por fractura proximal de tibia con limpieza quirúrgica y colocación de fijador de Taylor, sin embargo, a los 15 días se identificó necrosis del tejido con pérdida posterior del colgajo clasificándose como fracaso terapéutico. Todos los casos reportados fueron
Cuadro 1. Características sociodemográficas de los pacientes sometidos a reconstrucción por defecto de partes blandas, Servicio de Ortopedia y Traumatología, HE, 2012-2015, n=16.

\begin{tabular}{lc}
\hline Características & $\mathbf{n}(\%)$ \\
\hline Edad (años) & \\
$18-30$ & $6(37.5)$ \\
$31-40$ & $5(31.3)$ \\
$41-50$ & $1(6.3)$ \\
$51-60$ & $3(18.6)$ \\
$61-70$ & $1(6.3)$ \\
Promedio & 38.0 \\
Rango & $18-63$ \\
Sexo & \\
Masculino & $14(87.5)$ \\
Femenino & $2(12.5)$ \\
Ocupación & \\
Agricultor & $6(37.5)$ \\
Mecánico & $5(31.3)$ \\
Ama de casa & $1(6.3)$ \\
Operario & $1(6.3)$ \\
Estudiante & $1(6.3)$ \\
Pintor & $1(6.3)$ \\
Zapatero & $1(6.3)$ \\
Departamento de procedencia & \\
Francisco Morazán & $12(75.0)$ \\
Choluteca & $2(12.4)$ \\
Olancho & $1(6.3)$ \\
El Paraíso & $1(6.3)$ \\
Ambiente de procedencia & \\
Urbano & $12(75.0)$ \\
\hline Rural & $4(25.0)$ \\
\hline
\end{tabular}

seguidos hasta su egreso hospitalario, ninguno presentó complicaciones del colgajo posterior al egreso, siendo la estancia posquirúrgica entre 15 días o más (promedio 20.3 días). Se obtuvo carta aval del Servicio de Ortopedia y Traumatología del HE para llevar a cabo este reporte de casos.

\section{DISCUSIÓN}

La indicación del uso de colgajos se determina por la necesidad de cubrir una estructura profunda que no puede soportar la exposición al aire debido a la desecación, necrosis e infección consecuente; por lo que es conveniente, en un primer momento, evaluar la pérdida de sustancia y la estructura expuesta. ${ }^{9,10}$ Los músculos gastrocnemio y sóleo son muy utilizados en la reconstrucción del tejido blando en las fracturas abiertas de tibia que necesitan cobertura con colgajos; así, el gastrocnemio se selecciona para cubrir defectos en el tercio superior y medio; y el sóleo para el tercio medio y en ocasiones en el tercio 
Cuadro 2. Factores de riesgo/comorbilidades en pacientes sometidos a reconstrucción por defecto de partes blandas, Servicio de Ortopedia y Traumatología, HE, 2012-2015, $n=16$.

\begin{tabular}{lc}
\hline Factores de Riesgo & $\mathbf{n}(\%)$ \\
\hline Antecedentes personales patológicos & $13(81.1)$ \\
Negativos & $1(6.3)$ \\
Bocio & $1(6.3)$ \\
Hipertensión arterial & $1(6.3)$ \\
Diabetes mellitus & \\
Hábitos tóxicos & \\
Tabaquismo & $2(12.5)$ \\
Si & $14(87.5)$ \\
No & \\
Alcoholismo & $2(12.5)$ \\
Si & $14(87.5)$ \\
No & \\
Factores de riesgo quirúrgico & $16(100.0)$ \\
Negativos & \\
Condición vascular & $15(93.7)$ \\
Excelente & $1(6.3)$ \\
Buena &
\end{tabular}

Cuadro 3. Características clínicas de las fracturas en pacientes con reconstrucción por defecto de partes blandas; Servicio de Ortopedia y Traumatología, HE, 2012-2015, $n=16$.

\begin{tabular}{lc}
\hline Características de la fractura & $\mathbf{n}(\%)$ \\
\hline Diagnóstico traumatológico & $11(68.7)$ \\
Fractura expuesta proximal de tibia & $2(12.4)$ \\
Fractura expuesta diafisaria de tibia & $1(6.3)$ \\
Fractura expuesta distal de fémur & $1(6.3)$ \\
Fractura expuesta segmentaria de tibia & $1(6.3)$ \\
Fractura cerrada proximal de tibia & \\
Diagnóstico etiológico/médico & $13(81.3)$ \\
Politraumatismo & $3(18.7)$ \\
Herida por arma de fuego & \\
Mecanismo de la fractura & $6(37.5)$ \\
Peatón atropellado & $6(37.5)$ \\
Accidente en motocicleta & $3(18.7)$ \\
Por arma de fuego & $1(6.3)$ \\
Accidente automovilístico & \\
Clasificación de la fractura* & $7(43.7)$ \\
GIII A expuesta & $8(50.0)$ \\
GIII B expuesta & $1(6.3)$ \\
\hline Cerrada &
\end{tabular}

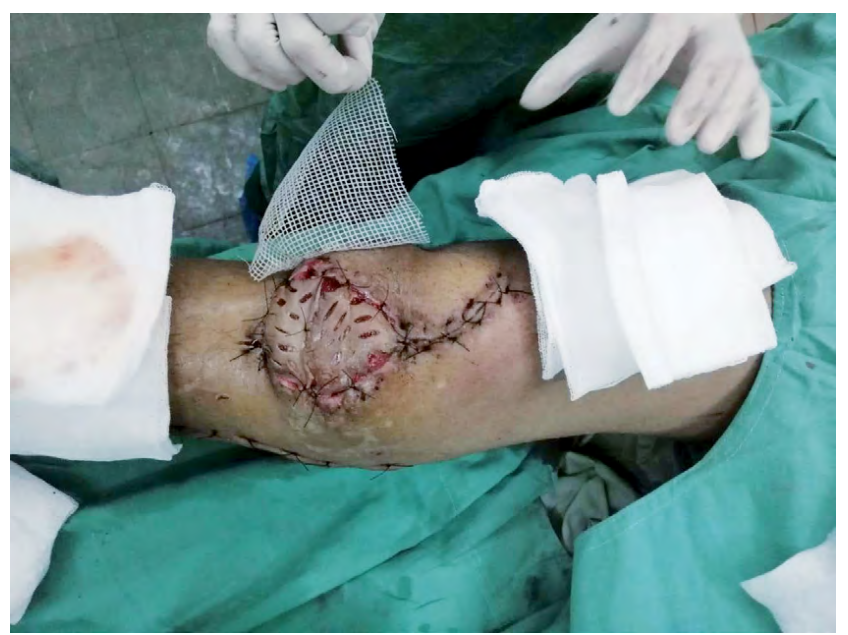

Figura 1. Reconstrucción total con colgajo Gastrocnemio en paciente atendido con Fractura proximal de tibia en $\mathrm{HE}$.

medio inferior de la tibia, ${ }^{9}$ siendo este uno de los recursos más utilizados debido a los bajos costos, y dificultad de disponer el personal especializado en reconstrucción de tejido, por lo que el cirujano ortopédico es el indicado para llevar a cabo este procedimiento. ${ }^{1-3,8}$ Esta técnica es utilizada en los casos de fracturas expuestas de miembros inferiores en el HE desde hace más de una década, sin embargo, de manera muy reciente se logró recolectar los casos tratados mediante este abordaje quirúrgico, demostrando el éxito del procedimiento, específicamente la disminución del riesgo de complicaciones que incluye la amputación el miembro afectado.

Un estudio prospectivo de intervención en pacientes con diagnóstico de osteomielitis crónica, en quienes se realizó colgajos musculares pediculados que incluyó 11 pacientes, demostró que la edad más frecuente osciló entre los 38 años, siendo todos del sexo masculino; de éstos, el 54.5\% (6/11) presentó fractura abierta, y concordaron, junto a Fernández Abreu \& Cols, ${ }^{11}$ respecto al éxito de la reconstrucción con colgajo muscular para el manejo de estos pacientes..$^{10}$ Datos que se asemejan a los presentados en nuestra serie de casos, en donde el promedio de edad fue de 38 años (rango 18-63) y siendo predominante el género masculino.

Entre los factores relacionados con riesgo de fracaso del colgajo se encuentran la edad avanzada, hipertensión arterial, diabetes mellitus, enfermedad venosa o tromboembólica, tabaquismo crónico y desnutrición. ${ }^{12-15}$ Antecedentes que en esta serie de casos solo se encontró un caso de bocio, hipertensión arterial y diabetes mellitus respectivamente. Así también, solo en $12.5 \%$ (2) casos se encontraron hábitos tóxicos de alcoholismo y tabaquismo. Pese a ello, no se encontró complicaciones directamente relacionadas con la colocación de los colgajos en estos pacientes que tenían factores de riesgo. 
Las fracturas abiertas de tibia y/o peroné tipo IIIB-IIIC de Gustilo son ocasionadas por traumatismos de alta energía tales como precipitaciones, accidente de tráficos y/o heridas por arma de fuego; y producen pérdida crítica de partes blandas y periostio (cobertura de tibia especialmente vulnerable), pérdida de hueso y extensa vascularización de la zona afectada. ${ }^{6} \mathrm{El}$ objetivo terapéutico de las fracturas expuestas se basa en conseguir una reposición adecuada y en el menor tiempo posible la cobertura externa para obtener una herida estable, promover la consolidación ósea, prevención de la infección, desbridamiento precoz, reconstrucción de todos los tejidos lesionados y vascularizados junto con la correcta estabilización y fijación ósea. ${ }^{6}, 16$ En esta serie de casos, los diagnósticos traumatológicos más frecuentes fueron las fracturas expuestas proximales de tibia en $68.7 \%$ (11), siendo la etiología el politraumatismo en $81.3 \%$ (13), mecanismo de fractura peatón atropellado y accidente en motocicleta $37.5 \%$ (6), así como fractura expuesta Grado IIIA y IIIB en $93.7 \%$ (15), en éstos se realizó un adecuado manejo de la fractura según el tipo de ésta ya sea con colocación de fijador externo, interno, de clavo endomedular bloqueado y/o inmovilización, y como manejo secundario la limpieza quirúrgica, además de la colocación de un colgajo muscular de sóleo y/o gastrocnemio de acuerdo a las características clínicas de la lesión.

Las fracturas abiertas de tibia complicadas con defectos o pérdida de tejido blando que se clasifican como Gustilo tipo IIIB o IIIC suelen ser de trauma sustancial que involucra lesiones complejas de hueso y tejido blando; la estabilización de la fractura y reconstrucción de tejidos blandos contribuye a salvar la función y supervivencia de las extremidades, por lo que deben ser desbridadas en múltiples ocasiones, hasta lograr una herida de apariencia limpia y sin signos locales de infección. ${ }^{17}$ Si la herida se deja abierta por un tiempo mayor a dos semanas existen altas probabilidades de riesgo de colonización y, por consiguiente, infección por la flora hospitalaria, así como un alto riesgo de fracaso terapéutico relacionado con el colgajo. ${ }^{10}$ En esta descripción solamente en un caso se encontró fracaso terapéutico por necrosis del colgajo comparado con la mayoría de los casos (15) en quienes no se presentaron complicaciones; probablemente relacionado a que se realizó la intervención quirúrgica en un tiempo promedio de 15 días en todos los casos.

Entre las complicaciones en estos pacientes se han descrito las asociadas a infección y relacionadas con la falla del colgajo, compresión de la vena poplítea u oclusión de ésta, contracción muscular involuntaria, cronicidad voluntaria de granulación del área para mantener un acceso directo a la administración de drogas o del sitio de origen del injerto, ${ }^{18-21}$ pero entre las más difíciles de manejar a mediano y largo plazo están las neuropatías relacionadas con compresión del nervio y relacionadas con neuropatía del peroné y que se presentan entre $8-19 \%$ de los pacientes, aunque se atribuye a la cirugía per se en el $25 \%$ de los casos, y el resto al trauma inicial. ${ }^{1,18}$ Otras complicaciones relativamente raras incluyen falta de cierre primario del sitio del donador con resultado cosmético desfavorable, hematoma del compartimiento superficial posterior de la pierna y contracción persistente del colgajo en el sitio donante ${ }^{; 2,13,21}$ evidenciando en algunas comunicaciones fallo del colgajo por estenosis de alto grado de la arteria sural. ${ }^{12}$ En este reporte de 16 casos solo se presentó una complicación asociada al procedimiento quirúrgico la cual fue necrosis del colgajo en un paciente sin factores de riesgo.

El uso de un colgajo gastrocnemio medial ha sido aceptado como procedimiento estándar que resuelve las alteraciones derivadas por deficiencia de tejidos blandos en torno al uso de prótesis, la mala irrigación sanguínea del colgajo muscular y la curación tendinosa. ${ }^{14}$ Es importante mencionar que estas lesiones conllevan siempre un manejo complejo, con gran consumo de recursos, y son susceptibles de generar morbilidad asociada con diferentes grados de discapacidad en un alto porcentaje de los pacientes tratados $;{ }^{16}$ es decir, el flujo venoso deficiente dentro del área contribuye a la presencia de enfermedades crónicas que puede provocar hipertensión venosa y ulceraciones posteriores lo que ha mostrado aumento en las tasas de osteomielitis que con frecuencia resultan en amputación de la extremidad. ${ }^{22}$ En vista de esto, entre los hallazgos de esta serie de casos se logró demostrar que con este procedimiento terapéutico se obtuvo buenos resultados y con mínimos recursos, entre estos la prevención del riesgo de complicaciones (1/16), prevención de riesgo de infecciones (ninguna) y salvamiento del miembro afectado (ningún caso fue sometido a amputación).

Uno de los principales aportes de esta descripción de serie de casos fue generar información sobre el éxito de la experiencia con la reconstrucción de tejido realizada por el cirujano ortopeda, con el uso mínimo de recursos llevada a cabo en la institución en un período de 4 años.

\section{CONTRIBUCIONES}

Todos los autores contribuyeron en la realización, redacción, atendieron las recomendaciones editoriales y aprobaron la versión final del artículo.

\section{AGRADECIMIENTOS}

Al Dr. Manuel Bueso por su apoyo y guía para la presentación de esta serie de casos, Hospital Escuela, Tegucigalpa, Honduras.

\section{DETALLES DE AUTOR(ES)}

José Nicolas Rodríguez Lara, Médico Especialista en Ortopedia y Traumatología; jose.rodriguez.85@hotmail.com

Mirna Patricia Ochoa Fletes, Médica Especialista en Ortopedia y Traumatología; ochoa.mirna.8@gmail.com 


\section{REFERENCIAS}

1. Parrett BM, Matros E, Pribaz JJ, Orgill DP. Lower extremity trauma: trends in the management of soft-tissue reconstruction of open tibia-fibula fractures. Plast Reconstr Surg [Internet]. 2006 [citado 2 marzo 2015];117(4):13151322. Disponible en: http://www.ncbi.nlm.nih.gov/entrez/query.fcgi? $\mathrm{cmd}=\mathrm{R}$ etrieve\&db=PubMed\&dopt=Citation\&list_uids $=16582806$

2. Masquelet A-C, Sassu P. Gastrocnemius flap. In: Fu-Chan Wi, Mardini S, editors, Flaps and reconstructive surgery. [Internet]. London, England: Elsevier; 2016. [citado 18 enero 2017]. p. 409-421. Disponible en: https:// www.elsevier.com/search-results?labels=books\&query=Flaps $\% 20$ and $\% 20$ Reconstructive $\% 20$ Surgery $\% 20 \&$ page $=1$

3. Noszczyk BH. The origins of the concept of muscle flaps. Br J Plast Surg [Internet]. 1996 [citado 18 enero 2017];49(2):107-110. Disponible en: http:// www.ncbi.nlm.nih.gov/pubmed/8733350

4. Zeiderman MR, Pu LLQ. Contemporary reconstruction after complex facial trauma. Burns Trauma [Internet]. 2020 [citado 11 agosto 2021];8: tkaa023. Disponible: https://pubmed.ncbi.nlm.nih.gov/32341916/

5. Rezende MR, Rabelo NTA, Wei TH, Mattar Junior R, de Paula EL, Zumiotti AV. Skin coverage of the middle-distal segment of the leg with a pedicled perforator flap. J OrthopTrauma [Internet]. 2010 [citado 6 marzo 2015];24(4):236-243. Disponible en: https://pubmed.ncbi.nlm.nih. gov/20335758/

6. Delgado Pérez JA, Rodríguez P, Liette MD, Masadeh S. Medial hemisoleus flap for middle third of the tibia defects. Clin Podiatr Med Surg [Internet]. 2020 [citado 11 agosto 2021];37(4):621-630. Disponible en: https:// pubmed.ncbi.nlm.nih.gov/32919594/

7. Pu LL. Soft-tissue coverage of an extensive mid-tibial wound with the combined medial gastrocnemius and medial hemisoleus muscle flaps: the role of local muscle flaps revisited. J Plast Reconstr Aesthet Surg [Internet]. 2010 [citado 6 marzo 2015];63(8):e605-e610. Disponible: https://pubmed. ncbi.nlm.nih.gov/20627760/

8. Baudet J, Pelissier P, Casoli V, Caix P, Farias FJ, De Pawlikowski W. Soleus flap. In: Wei FC, Mardini S. Flaps and Reconstructive Surgery [internet]. $2^{\mathrm{a} e d .}$ Londres: Elsevier; 2016. [citado 10 enero 2017]. p. 423-438. Disponible en: https://www.elsevier.com/searchresults?labels=books\&query=Flaps $\% 20$ and $\% 20$ Reconstructive $\% 20$ Surgery $\% 20 \&$ page $=1$

9. Masquelet AC. Lambeaux pédiculés des membres. Techniques chirurgicales-Orthopédie-Traumatologie. [Internet]. Paris: Elsevier Masson SAS; 2021. [citado 10 abril 2021]. Disponible en: https://www.elsevier. com/search-results?labels=books\&query=Techniques $\% 20$ chirurgicalesOrthop \%C3\%A9die-Traumatologie\&page $=1$

10. Salles Betancourt GR, Croas FA. Rotación de colgajos musculares para cobertura de defectos en fracturas abiertas de tibia producidas por armas de fuego. Rev Cubana Ortop Traumatol [Internet]. 2010 [citado 5 febrero 2019];24(1):1-11. Disponible en: http://scielo.sld.cu/scielo.php?pid=S0864215X2010000100004\&script=sci_arttext.

11. Fernández Abreu AF, Santana López $P$, Chiong Castillo $M$, Ducongé Oliva D, Fernández Breu SM. Empleo del Colgajo Muscular Sóleo como cobertura de los defectos cutáneos. Rev Cubana Med Milit [Internet]. 2002 [citado 5 febrero 2019];31(3):209-14. Disponible en: http://scielo.sld.cu/pdf/mil/ v31n3/mil09302.pdf

12. Hedley D, Munnoch DA, Hancock K. Pre-existing venous disease and the gastrocnemius muscle flap. Br J Plast Surg [Internet]. 2001 [citado 10 marzo 2017];54(6):559-560. Disponible en: https://pubmed.ncbi.nlm.nih. gov/11513534/

13. D'Avila F, Franco D, D'Avila B, Arnaut M. Use of local muscle flaps to cover leg bone exposures. Rev Col Bras Cir [Internet]. 2014 [citado 2017 marzo 10];41(6):434-9. Disponible en: https://pubmed.ncbi.nlm.nih. gov/25742410/. DOl:10.1590/0100-69912014006009.

14. Kuo FC, Jeng SF, Lin TP, Wang JW. Gastrocnemius musculotendinous flap for reconstruction of extensor mechanism after proximal tibial tumor resection. Formosan J Musculoskeletal Disord [Internet]. 2011 [citado 25 septiembre 2017];2(1):31-34. Disponible en: https://www.sciencedirect. com/science/article/abs/pii/S2210794010000374

15. Castillo DP. Colgajos musculares y musculocutáneos: Conceptos generales. Cuad Cir [Internet]. 2003 [citado 10 mar 2017]; 17:71-74. Disponible en: http://revistas.uach.cl/pdf/cuadcir/v17n1/art12.pdf

16. Camporro Fernández D, Ontaneda Rubio A, Castellanos Morán M. Tratamiento de fracturas abiertas de tibia grado IIIB-IIIC de Gustilo con colgajos libres microvascularizados. Cir. Plást Iberolatinoam [Internet]. 2015 [citado 10 marzo 2017];41(3):283-293. Disponible en: https://scielo.isciii.es/scielo. php?script=sci_abstract\&pid=S0376-78922015000300008

17. Jitprapaikulsarn $\mathrm{S}$, Benjawongsathien $\mathrm{K}$, Patamamongkonchai $\mathrm{C}$, Gromprasit A, Thremthakanpon W. Combined medial gastrocnemius and hemisoleus flap: a reproducible alternative for open tibial fractures complicated with large or double soft tissue defects. Eur J Orthop Surg Traumatol [Internet]. 2021 [citado 202111 agosto];31(2):413-20. Disponible en: https:// pubmed.ncbi.nlm.nih.gov/32808120/

18. Sanger JR, Kao DS, Hackbarth DA. Peroneal nerve compression by lateral gastrocnemius flap. J Plast Reconstr Aesthet Surg [Internet]. 2009 [citado 10 marzo 2017];62(8): e280-282. Disponible en: https://pubmed.ncbi.nlm. nih.gov/18222740/

19. Mena Pérez R, Murciano Guerra RF, González González P. El colgajo muscular pediculado como variante de tratamiento en la osteomielitis crónica. Rev Cubana Ortop Traumatol [Internet]. 2009 [citado 5 febrero 2019];23(1):1-17. Disponible en: http://scielo.sld.cu/scielo.php?script=sci_ arttext\&pid=S0864-215X2009000100006.

20. Iyer S, Pabari A, Khoo CT. A well vascularised muscle flap--drug user's dream. J Plast Reconstr Aesthet Surg [Internet]. 2012 [citado 5 febrero 2019];65(3):399-401. Disponible en: http://www.ncbi.nlm.nih.gov/pub$\operatorname{med} / 21873130$.

21. Baser NT, Barutcu AY, Isik V, Aslan G. Closure or reduction of the donor defect of a sural flap with a purse-string suture: long-term results. J Plast Surg Hand Surg [Internet]. 2011[citado 5 febrero 2019];45(6):267-273. Disponible en: http://www.ncbi.nlm.nih.gov/entrez/query.fcgi?cmd=Retrieve\& $\mathrm{db}=$ PubMed\&dopt=Citation\&list_uids $=22250718$

22. Fisal AA, Romeih MAH, Younes LM, El-Rosasy M, Rodrìguez P, Liette MD, et al. Distally based medial hemisoleus muscle flap for wound coverage in the distal third of the leg. Clin Podiatr Med Surg [Internet]. 2020 [citado 11 agosto 2021];37(4):631-647. Disponible en: https://pubmed.ncbi.nlm.nih. gov/32919595/

ABSTRACT.Background: Bone exposure can be the consequence of open or closed traumatic fractures with complications, as well as secondary to infectious processes. The coverage of the lower limb with a skin defect is based on the preservation of blood and nerve perfusion to promote bone consolidation and thus the salvage of the limb. Clinical case description: The reconstruction with a pedunculated rotational muscle flap of the soleus and/ or gastrocnemius is described in 16 patients with complications associated with exposed fractures in the lower limbs with soft tissue defects and loss of skin coverage, performed in the Orthopedics and Traumatology at the Escuela Hospital during the period 20122015. A soleus flap was placed in $50.0 \%$ (8/16) and gastrocnemius in $50.0 \%(8 / 16)$. Surgically and functionally qualified therapeutic success was $87.5 \%$ (14/16). Conclusion: With the reconstruction technique performed on these patients, it was possible to reduce the frequency of complications and therefore avoid amputation of the affected limb.

Keywords: Honduras, Muscles, Orthopedics Surgical Flaps. 